\title{
Investigating Mathematics Trainee Teachers' Conceptions of Proof Writing in Algebra: A Case of One College of Education in Zambia
}

\author{
Kenneth M. Likando \\ Department of Mathematics Education, University of South Africa (UNISA), \\ PO Box 392 Pretoria 0003 \\ Email: likandomk@yahoo.com \\ Mapula G. Ngoepe \\ Department of Mathematics Education, University of South Africa (UNISA), \\ PO Box 392 Pretoria 0003 \\ Email: ngoepmg@unisa.ac.za
}

\section{Doi:10.5901/mjss.2014.v5n14p331}

\begin{abstract}
This paper investigates mathematics trainee teachers' conceptions of proof writing in algebra at Copperbelt College of Education in Zambia. The ability to construct proofs seems to be an important skill for all mathematicians and those who have interest in mathematics education. However, literature shows that proof is widely acknowledged to be one of the most difficult aspects in the mathematics curriculum. The sample comprised of 35 male and 15 female third year mathematics trainee teachers. Data was collected by means of a self-administered questionnaire, a written diagnostic test and oral interviews. The three instruments were used to provide multiple views to triangulate the data. The results show that students seem to understand proof as a verification and confirmation tool. Many of them applied empirical approaches in their written proofs. They did not have sufficient knowledge of the right set of accepted statements such as axioms and could not identify which statements required proofs in mathematics. These findings suggest that students' conceptions of proof were underdeveloped hence affecting their ability to produce valid proofs. These findings will assist in guiding curricula designers and teachers of proof related knowledge.
\end{abstract}

Keywords: algebra; trainee teachers; proposition; Zambian curriculum; conceptions of proof

\section{Introduction}

Experience shows that despite exposing students to the necessary pre-requisite knowledge before learning proof; and the application of varied methods of teaching, students do not seem to show any significant improvements in proving mathematical hypotheses. The misconceptions that students have in writing proofs are probably compounded by the fact that in the Zambian curriculum for secondary mathematics there appears to be a huge gap between the school syllabus and the college. The school syllabus far falls short of the necessary pre-requisite knowledge that could enhance the development of proof skills in the college. This denies school leavers of the much needed smooth transition to college proof experiences later. This is despite the fact that the concept of proof forms a vital integral part of the syllabus in the training of mathematics teachers in colleges and universities in Zambia.

According to the National Council of Teachers of Mathematics NCTM (2000), a mathematical proof is 'a formal way of expressing particular kinds of reasoning and justification' (NCTM, 2000, p56). By the end of high school, a student is expected to be in a position to make use of mathematical reasoning to "produce mathematical proofs, and should appreciate the value of such arguments" (NCTM, 2000, p56). Being able to reason is critical in the learning of all mathematics from the early years of one's school life (Weber, 2010). This seems to suggest that some form of reasoning and proof should have a place in school mathematics curriculum at all levels in order to consolidate proof conceptions and critical thinking skills. This is unfortunately not the case in Zambia where proof is first met after high school.

There is sufficient literature of research done on students' difficulties in proof. Senk (1989) conducted a study in which proof-writing skills were tested among 1520 students in five states of the United States of America. Weber (2001:101) also investigated students' difficulty in constructing proofs in abstract algebra. However, the extent to which students' conceptions of proof influenced their ability to construct algebraic proof has not been investigated. This is where 
this paper fills in the gap. Therefore, the research question that this paper attempts to answer is: To what extent do students' conceptions of proof influence their ability to construct algebraic proofs?

\section{Literature Review}

As proof is widely acknowledged to be one of the most difficult aspects of the mathematics curriculum (Clements \& Battista, 1992; Moore, 1994; De Villiers, 2004), it is important that we understand how concepts and skills related to proof could be developed. Two predominant theories about the development of reasoning and proof are associated with the works of Piaget and van Hiele. Piaget describes how the development of proof occurs without considering curricula, while van Hiele analyses progress in proof abilities with curricula. However, both views provide insight into how students can develop conceptions of proof so that we are able to determine the level of reasoning at which they are operating. These theories are relevant in this study as they provide an understanding of how proof conceptions develop and could contribute to the correct interpretation of any peculiar findings in students' produced proofs.

In his early works of a child's genesis of thought, Piaget identified three stages inherent in concept formation. They include the first stage called perceptual, that is, when a child is not able to discover a concept concerning the subject in question. This means, a child completely fails to understand a problem (van Hiele 1959, p12). The second stage called conceptual involves knowing something about the concept but failing to make use of it. Here a child is easily misled by external circumstances (van Hiele1986, p11). Moore (1994, p254) provides interesting findings related to this stage. He closely observed five undergraduate students as they progressed through an introductory proof course. Moore found that students could sometimes state the definition of a concept with very little understanding of it but failed to see the relevance of using such concepts in proofs. He describes this as lack of concept images for doing proofs. According to van Hiele, this kind of thinking is reminiscent of a child's thinking process at the second stage of his thinking levels.

The third stage is the abstraction where a child knows reasonably well how to operate with the concept (van Hiele, 1986, p11). Abstractions involve developing appropriate conceptions of axioms and definitions which eventually culminate into theorems at different stages. At this stage a learner is capable of understanding proof. Nixon (2005:22) notes that concepts acquisition that links percepts to abstractions is vital in fostering the understanding of proof.

Van Hiele's original research constituted of five levels of thinking which were based on geometry though he later (van Hiele 1996) acknowledged that these levels could be applied to algebra as well. Recently, Land (1990) and Nixon (2005) successfully carried out studies in which they adapted van Hiele levels of thinking to algebra.

Many studies (for example, Senk, 1989; Land, 1990; De Villiers, 2003 and Nixon, 2005) suggest that deductive reasoning and initial proof abilities first occur at level three of the van Hiele hierarchy because this is the time when the network of logical relations between properties of concepts is established. In addition, De Villiers has observed that a prolonged delay at van Hiele Levels 1 and 2 before introducing proof could cause students to look at proof as a more difficult activity. Other studies, for example, (Usiskin, 1982; Healy \& Hoyles, 2002; Gibilisco, 2005) point to abrupt introduction of formal proofs in colleges as one of the major causes of students' difficulty in understanding proof. As pointed out earlier, this could be true in the case of Zambia where proof is first introduced at college or university.

The theories of van Hiele and Piaget in Land (1990) and Nixon (2005) on the development of reasoning and proof as well as their inherent levels of thinking form the theoretical base for this study. In particular, van Hiele's levels of thought are vital and relevant to this study as they provide an understanding of how students develop thinking conceptions as they progress through the different levels of thinking under the influence of school curriculum. These conceptions largely affect their ability to master formal mathematical proofs (Senk, 1989).

De Villiers (2003) and van Hiele (1996) believe that a child who has not yet achieved an appropriate level of thinking for understanding proof is unlikely to cope with instruction at that level. As this study is on students' conceptions of proof writing in algebra, van Hiele's third and fourth levels played a critical role because of the evidence from literature that it is at level three where appropriate behaviours pertaining to comprehension of proof begin to emerge so that by the fourth level students would have developed more proficiency in proof.

Selden and Selden (2003) carried out a research to determine whether undergraduate students were able to validate algebraic proofs as correct proofs of the given theorems. The findings revealed that students had a tendency to focus on the surface features of arguments and that their ability to determine whether arguments were proofs were very limited.

Mason in Bednarz (1996) links the difficulties students have in proof-writing to their misconceptions in algebra where most linguistic difficulties relate to variables and expressions. This is acknowledged by Grouws (1992) who suggests that some of the misconceptions that students have in proof seem to result from over-generalising or from combining pieces of information in inappropriate ways. Another misconception is that students find it difficult to recognize 
a general proof as complete without testing empirical examples. This could as well be ascertained from the works of Healy and Hoyles (2002) in their study of proof misconceptions among British students which revealed that the majority of them were unable to construct valid proofs in the domain of algebra because most of them had a misconception that general and explanatory arguments constituted proof.

These findings are useful to this study as they provide a link between the difficulties students have and their illunderstanding of the value of proof in mathematics. Further, the findings will provide a genre of knowledge that will act as a springboard for explanations of certain behaviours that will arise in the study on the extent to which students' conceptions of proof influence their ability to construct algebraic proofs.

To better understand students' conceptions of proof, it may be necessary to study the processes that students engage in as they construct algebraic proofs.

\section{Research Methodology}

\subsection{Sample}

Qualitative research approach was used to shed light on students' conceptions of proof and how these relate to their ability to construct algebraic proofs. The participants were third year mathematics trainee teachers (35 male and 15 female). This cohort of students was suitable because they had already been exposed to most of the preliminary topics for proof.

\subsection{Instruments}

Three instruments were used in the collection of data. These were a self-administered semi-structured questionnaire, a diagnostic test (written test) and oral interviews. The questionnaire items were grouped in themes and categories that best represented the main idea in the items in relation to the research question. All the fifty students participated in the answering of the questionnaire.

The written test comprised four tasks on proof-writing given to a focus group of six third-year mathematics students in order to provide an in-depth test to discover the nature of particular weaknesses, difficulties and conceptions that students possess in proof construction (Cohen et al., 2000). The test items were familiar to the students but they were not exclusively of a routine type as those they met in the classroom or textbooks. This was done to avoid the possibility of reproducing memorised arguments. Each test item was structured in three-open ended parts with part (a) asking them to define the underlined key word in the proposition. Part (b) requested them to write two examples of the proposition and (c) requested them to prove the proposition showing details of their working.

The use of interviews in this study was motivated by Stillman and Galbraith $(1998$,$) who contend that interviews$ are useful for investigating students' mathematical thinking and are the most appropriate method for the discovery of cognitive processes and the evaluation of competence. Another advantage of interviews is provided by Maluku and Sanders (1994) who postulate that during the process of an interview a researcher can probe interesting or incomplete responses allowing respondents to clarify or extend their answers. Each interview lasted approximately 30 minutes for each of the six students. During the interviews students were made to think aloud as they reflected on their experiences. The interview did not look at the surface of the given answers but he probed to find out what was behind the given answers that mattered. All the interview sessions were audio-taped and later transcribed verbatim for pattern matching analysis according to identified themes and categories in order to answer the research question.

The use of three different methods of data collection was useful for triangulation purposes. The benefits of triangulation include "increasing confidence in research data, creating innovative ways of understanding a phenomenon, revealing unique findings, challenging or integrating theories, and providing a clearer understanding of the problem" (Thurmond 2001, p 254).

Responses to the questionnaire, the test and the interview were analysed to make sense out of the collected data. Three basic steps of organizing the data collected, describing the relevant aspects of the study and interpretations of the data (Creswel, 2003) were employed. Data was interpreted to bring out lessons that were learnt. The findings of the analysis were conveyed through a detailed study of the themes and categories supported by quotations and specific evidence from data. Graphical and tabular presentations were occasionally used in an attempt to cross-validate, confirm or corroborate findings. 


\section{Findings and Discussions}

This section focuses on the findings to answer the question: Do students' conceptions of proof influence their ability to construct algebraic proofs? Students were asked to provide written descriptions about proof and what they thought it was in the questionnaire. The question read as follows:

\subsection{Explain your understanding of what proof is in mathematics.}

From the analysis of the fifty (35 males and 15 females) students' explanations of their understanding of what proof is in mathematics, a total of four categories emerged. Table 1 below provides a summary of the three categories of students' understanding of what proof is in mathematics.

Table 1. Students' explanations of their understanding of the concept of proof

\begin{tabular}{|c|c|c|}
\hline \multicolumn{1}{|c|}{ Categories } & $n=50$ & Percentage \\
\hline Verification of correctness of mathematical results (using specific numbers) & 37 & $74 \%$ \\
\hline A valid chain of deductive reasoning (using definitions, axioms,...) & 10 & $20 \%$ \\
\hline No response (i.e. relevant answer spaces left blank) & 3 & $6 \%$ \\
\hline Total & 50 & $100 \%$ \\
\hline
\end{tabular}

From the table above, the majority of the students $(74 \%)$ were of the opinion that a mathematical proof constitutes verification of the truth of a mathematical statement. This could be interpreted to mean that most of the students' understanding of the concept of proof is largely empirical. That is, many students believe that verifying that a theorem holds in a specific instance or several instances is sufficient proof. This implies that students do not really seem to understand what it means to prove something. Similar results emerged during their written proof and the oral interviews where four of the six students expressed the opinion that by replacing numbers in the proposition a proof is accomplished. Examples of these are found in the "proofs" of propositions 1 and 2 at pages 13 and 14. These misconceptions were not surprising as similar results have been found in other studies (for example, Weber 2001; De Villiers 2003; Nyaumwe \& Buzuzi, 2007).

Table 1 findings further reveal that only $20 \%$ of the respondents believed that proof consisted of valid chains of deductive reasoning that involve use of definitions and axioms. This small percentage point is an indication that the majority of the students have a wrong conception of what constitutes proof in mathematics.

The next item required students to write down any algebraic mathematical statement (or theorem) that would need proof. The question read as follows.

\subsection{Write down any mathematical statement (proposition) that would need proof.}

Students' written statements were analysed and categorised according to whether the statement was suitable for proof, not suitable for proof or none. Table 2 provides these findings.

Table 2. Categories of algebraic mathematical statements produced by students

\begin{tabular}{|l|c|c|}
\hline \multicolumn{1}{|c|}{ Categories } & \multicolumn{2}{c|}{ Number of respondents $(\boldsymbol{n}=\mathbf{5 0})$} \\
\hline Valid (Suitable for proof) & 30 & $60 \%$ \\
\hline Invalid (Not suitable for proof) & 16 & $32 \%$ \\
\hline No response (i.e. relevant answer spaces left blank) & 4 & $8 \%$ \\
\hline Total & 50 & $100 \%$ \\
\hline
\end{tabular}

The table above shows that 30 of the 50 respondents were able to write down valid statements which were suitable for mathematical proof. Sixteen respondents provided invalid statements and four of them did not give any response at all. Of the thirty valid propositions provided by students, some of the examples included the following statements:

The sum of two odd numbers is even

The product of two even numbers is even. 
The square root of two is irrational (that is, square root of two cannot be expressed in the form $a / b$ where $a$ and $b$ are relatively prime).

$1+2+3+\ldots+n=1 / 2 n(n+1)$.

This item could also be linked to the one the six students responded to during the oral interviews. For example, the following excerpt provides more information on the verbatim responses provided:

(With reference to Proposition 1: For any positive integer $n$, if $n^{2}$ is even, then $n$ is even)

Interviewer: Can you state another statement that is related to the one given?

Eric: If I have $2 n$, I square it, I still get an even number where $n$ is any number from the set of rational numbers.

Jane: The product of two even numbers is even.

Peter: If $n$ is an odd number, then $n 2$ is odd.

Only three of the six respondents attempted to answer this item. As seen from the above responses, Eric starts with $2 n$, which is a general form of an even number, which when squared $(2 n)^{2}$ results into another even number. Thus, if we let the even number $2 n$ to be equal to $x$, we can re-write Eric's statement as: "If $x$ is even, then $x^{2}$ is even." This is true because the relationship from the proposition is an example of a necessary and sufficient condition, which is, $x^{2}$ is even if and only if $x$ is even. However, Eric describes $n$ in $2 n$ to be from the set of irrational numbers which is flawed. For example, if $n$ is the irrational number $1 / 2$ then $2 n$ which is supposed to be even becomes 1 which is odd, which renders the statement wrong. Therefore, Eric's statement is simply a restatement or converse of the Proposition 1 where $n$ must necessarily be from the set of positive integers.

Peter's statement can be restated as: "If $x$ is odd, then $x^{2}$ is odd" which is the contrapositive of proposition 1. Similarly, the statement: "If $x^{2}$ is odd, then $x$ is odd" is the contrapositive of the implication: "If $x$ is even, then $x^{2}$ is even." Jane gave an interesting theorem in that the product of two even numbers will always yield an even product. In fact, Jane's statement could be a superset of proposition 1 as it is more general. The converse of Jane's statement is meaningless unless if the number referred to is one and the same.

Results from both the table and oral interviews suggest that a large number (40\%) of the respondents to the questionnaire and half from the interviews, do not possess sufficient knowledge of mathematical statements that require mathematical proof because they were unable to produce the valid related statements. This could also be linked to their lack of correct conception of proof as perceived in the first item of the questionnaire. Consequently, if students could not understand what proof was, it would possibly be difficult for them to know statements that needed proof in mathematics.

The third item in the questionnaire was meant to assess students' knowledge of axioms used in proving mathematical statements. Students were required to state any axiom that would be essential in proving the proposition they had stated in the second item above. The item read as follows:

\subsection{Write down any axiom that is essential in the proof for the statement you provided in Question 2.}

This item was meant to assess students' knowledge of axioms used in proving mathematical statements. Axioms help us in the construction of proofs and they themselves are statements that are taken as true and need no proof when used in a chain of deductive arguments (Gibilisco 2005:60). Each of the fifty respondents in the questionnaire was requested to suggest any axiom that they thought could be useful in constructing a proof of their own statements given in the second item. Responses were analysed to determine whether a suggested axiom was acceptable or not. The criteria used to determine the usefulness of the given axiom was determined by the researcher's knowledge of proof, use of available literature as well as consultation with other senior lecturers in the Department of Mathematics at the college. An axiom was considered acceptable if it belonged to the field of mathematics and it would be useful in the proof of the respective statement or theorem stated by the student. An axiom was considered unacceptable if it belonged to the field of mathematics but it would not be useful for the proof of the mathematical statement. An "axiom" was considered invalid if it was mathematically meaningless and did not make any sense at all. Some of the examples from student responses were: $2 n$ is even; $(2 n+1)$ is odd; 1 is the first counting number; $-1 X a=-a$; For any integer $a$, $a^{2}$ is positive. The categories derived are presented in Table 3. 
Table 3. Students' responses showing suggested axioms to be used in proving their own mathematical statements produced in item 2.

\begin{tabular}{|l|c|c|}
\hline \multicolumn{1}{|c|}{ Categories } & $\boldsymbol{n}=\mathbf{5 0}$ & Percentage \\
\hline Acceptable and useful axiom & 15 & $30 \%$ \\
\hline Unacceptable but not useful axiom & 7 & $14 \%$ \\
\hline Invalid axioms & 13 & $26 \%$ \\
\hline No response (i.e. relevant answer spaces left blank) & 15 & $30 \%$ \\
\hline Total & 50 & $100 \%$ \\
\hline
\end{tabular}

The results revealed that only $30 \%$ of the responses were considered as acceptable and useful axioms in the proof of the corresponding propositions. Fourteen percent of the responses were unacceptable as they could not be of any use in the proofs of the corresponding propositions. Interestingly, $26 \%$ of the respondents provided invalid axioms while a number of respondents (30\%) did not write anything. The total percentage of those who wrote either an invalid axiom or no response at all was $56 \%$. This represents the majority of the students. This could probably suggest that the majority of the students did not seem to even know the axioms that could be used in proving mathematical statements. Stylianides (2007) observes that, in a genre of mathematical proofs, axioms and definitions are vital in establishing mathematical arguments.

The findings in the foregoing discussion have clearly revealed that students seem to have misconceptions in understanding what proof is, the axioms used in proof and statements that need proof. These and many more misconceptions were further brought to light in the analysis of their written proofs in the test where a considerable number of them produced an empirical approach of argumentation. In their written "proofs", Carol, Eric, Peter and Thabo, produced empirical proofs, with hardly any axioms used. For example, Carol's "proof" in Figure 2 (below) attests to this fact. These findings seem to suggest that generally, students possess only limited knowledge of axioms to be applied in proofs of propositions. Even those who have sufficient knowledge of axioms tend to find it difficult to apply them correctly and appropriately to specific proofs.

\subsection{Written test (Students' written "proofs")}

The written test was one of the main research instruments in the study. It was a diagnostic tool to assess students' ability to define key concepts in a given proposition, to provide examples of a given proposition and to prove the algebraic proposition or theorem. The main purpose of the test was specifically to provide in-depth testing to discover the nature of conceptions that students exhibited in proof construction. Candidates were given four questions on proof out of which only two odd numbered questions, namely question 1 and 3 were sampled for the purpose of this report. In addition, these two were the most attempted and that a random check revealed that candidates wrote more on these two of the four propositions. The propositions were stated as follows:

Proposition 1: For any positive integer $\mathrm{n}$, if $\mathrm{n} 2$ is even, then $\mathrm{n}$ is even.

Proposition 2: The sum of two consecutive odd numbers is divisible by four.

For example, in Proposition 1 Carol and Eric and in Proposition 2 Carol, Peter and Thabo (these are pseudonyms meant to protect students' real identity) all produced empirical examples as proof. Their purported proofs consisted of one or several numerical examples offering confirmation or verification, yet incomplete evidence for the truth of the propositions. Eric's proof provides a typical representation of this kind of arguments.

Figure 1: Eric's "proof"

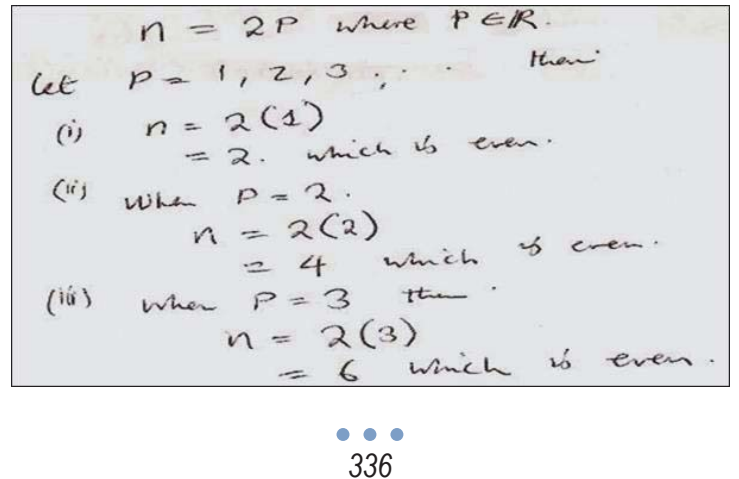


Eric's "proof" of proposition 1: For any positive integer $n$, if $n^{2}$ is even, then $n$ is even

\subsection{Analysis of Eric's "proof"}

In the first step:

[1] $n=2 p, p=1,2,3, \ldots$ where $p \in R$.

This representation is an appropriate way to start this proof. One can take it to mean: Let $n=2 p$ where $p$ is a positive integer. However, the fact that $p$ is being defined as a real number, $R$, in $2 p$ and at the same time as a positive integer in $p=1,2,3, \ldots$ creates some ambiguity. Besides, $n$ is not well defined as the set to which it refers is not stated, but one may probably assume that it is implied from the proposition the student author is attempting to prove. In proofs, it is advisable to clearly describe or define every variable used in order to avoid ambiguity.

In the second step:

[2] then (i) when $p=1, n=2(1)=2$ which is even. (ii) when $p=2, n=2(2)=4$ which is even. (iii) when $p=3, n=$ $2(3)=6$ which is even.

Like in the case of Carol, Eric took an empirical approach though he uses many instances (no matter how many specific cases one considers they will never prove a mathematical proposition or theorem). He fails to utilise concept definitions and other acceptable mathematical statements to reason with general deductive arguments. Apparent also in Eric's "proof" was the absence of a link in individual inferences to further his arguments. This was due to lack of a concept image for proof.

In proposition 2, Carol's proof provides another typical example of the other four.

Figure 2: Carol's "proof".

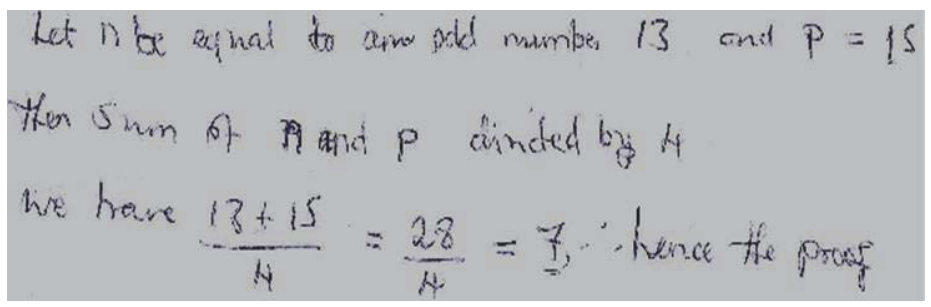

Figure 2. Carol's "proof" of proposition 2: The sum of two consecutive odd numbers is divisible by four

From Carol's "proof" in figure 2 it is clear that axioms were not used and the mode of argumentation was empirical as specific numbers were used to prove the truth of the proposition. The student seems to believe that non-deductive arguments constitute a proof. According to Stylianides and Stylianides (2007) use of an empirical mode of argumentation does not constitute proof in mathematics. These findings suggest that these students lack a correct conception of what constitutes proof in mathematics. Differently stated, students did not seem to possess sufficient 'concept images' for doing proofs. Tall and Vinner (2003) define the term concept image as the total cognitive structure that is associated with a concept. This includes all the mental pictures, associated properties and processes relating to the concept.

The foregoing findings could also be linked to van Hiele's theory on proof abilities which were referred to in the literature review. According to this theory, students begin to attain the development of proof abilities at level three (Land, 1990). While at level 4 students can construct proofs, understand the role of axioms and definitions, and know the definition of necessary and sufficient conditions. Consequently, it may be suggested in these results that students' inability to perform proof tasks satisfactorily could probably be attributed to their not having had attained these van Hiele levels which are so crucial in developing proof abilities (van Hiele, 1986).

\section{Conclusion and Implications}

Some of the significant inferences drawn here are that students seem to understand proof as a verification and confirmation tool. As a result, many of them applied empirical approaches in their written proofs. This was an indication that they lack conceptions of what constitutes a mathematical proof. The findings also revealed that students did not have sufficient knowledge of the right set of accepted statements called axioms, which are critical in establishing deductive 
arguments in mathematics. Furthermore, students seem to have problems identifying which statements required proofs in mathematics. These conceptions could have led to the nature of proofs that they wrote. Most of their proofs were either empirical in nature or lacked axioms and definitions that were vital in the production of valid deductive arguments required in a mathematical proof. These findings suggest that students' conceptions of proof were underdeveloped which compromised their ability to produce valid proofs.

The implications of the results obtained in this study on students' misconceptions of proof cannot be overstressed. The findings of this research could be subjected to other interpretations. We acknowledge that many issues surrounding students' conceptions in algebraic proofs may not have all been captured here. Regardless of these limitations, the findings of this study do still suggest some valuable implications that seem to have emerged.

It is recommended that lecturers, designers of curriculum and text book writers take into consideration these misconceptions students have so that designing of curricula and teaching of proof related knowledge are done in a manner that addresses them. It is further recommended that teachers and lecturers try as far as possible to relate proof to context instead of teaching it in abstract form so as to assist in the development of appropriate proof conceptions.

\section{References}

Bednarz, N., Kieran, C. \&Lee, L. (1996). Approaches to algebra: Perspectives for research and teaching. Dordrecht: Kluwer Academic Publishers.

Cohen, L. Manion, L \& Morrison, K. (2000). Research Methods in Education, $5^{\text {th }}$ edition. London: Routledge Falmer.

Creswell, JW. (2003). Research Design, 2nd edition. Thousands Oak: SAGE Publishers.

De Villiers, M. (2003). Rethinking for a proof with the Geometer's sketchpad. Emeryville: Key Curriculum Press.

Gibilisco, S. (2005). Math proofs demystified. New York: McGraw-Hill.

Grouws, DA (ed). (1992). Handbook of Research on Mathematics Teaching. New York: Macmillan Publishing Company.

Healy, L. \& Hoyles, C. (2002). A study of proof concepts in algebra. Journal for Research in Mathematics Education.13( 4), 396-448.

Land, J. E. (1990). Appropriateness of the van Hiele model for describing the student's cognitive process on algebra tasks as typified by college students' learning of functions. Boston: U.M.I.

Moore, R.C. (1994). Making the transition to formal proof. Educational Studies in Mathematics, 27, 249-266.

Narainsamy, S. (1998). The use of LOGO in the teaching of the concept of a variable in the pre-algebra stage. MEd Dissertation. Pretoria: UNISA.

National Council of Teachers of Mathematics [NCTM]. (2000). Principles and Standards for School Mathematics. Reston, VA: NCTM.

Nixon, E. G. (2002). An investigation of the influence of visualization, exploring patterns and generalization on thinking levels in the formation of the concepts of sequences and series. MEd Dissertation. University of South Africa, Pretoria.

Nyaumwe, L \& Buzuzi, G. (2007). Teachers' attitudes towards proof of mathematics results in the secondary school curriculum: The case of Zimbabwe. MathematicsEducation Research Journal. 19(3): 21-31.

Sanders, M. \& Mokuku, T. (1994). How Valid is Face Validity? Proceedings of the second Annual Meeting of the Southern African Association for Research and Development in Mathematics and Science Education. Durban, January: 479-489.

Selden A. \& Selden J. (2003). Validations of proofs considered as texts: Can undergraduates tell whether an argument proves a theorem? Journal of Research in Mathematics Education.34(1): 4-36.

Senk, S.L. (1989). Van Hiele levels and achievement in writing geometry Proofs. Journal for Research in Mathematics Education. 20(3): 309-321.

Stillman, G.A.\& Galbraith, P. L. (1998). Applying mathematical real world connections: metacognitive characteristics of secondary students. Educational studies in mathematics. 36, 157-195.

Stylianides, A. J. (2007). Proof and Proving in School Mathematics. Journal for Research in Mathematics Education.38(3): 289 -321.

Tall, D. \& Vinner, S. (2003). Concept image and concept definition in mathematics with particular reference to limits and continuity. The Montana Mathematics Enthusiast, 12, (2): 231- 248.

Thurmond, V.A. (2001). The point of triangulation. Journal of Nursing Scholarship. 2001; 33(3):253-8.

Usiskin, Z. (1995). Why is algebra important to learn? American Educator. 19(1):30-37.

Usiskin, Z. (1982). Van Hiele levels and achievement in secondary school geometry - CDASSG Project. Chicago: University of Chicago.

Van Hiele, P.M. (1996). P.M. van Hiele lectures during his visit to South Africa in 1996. Reader for MEDMA. Pretoria: UNISA.

Van Hiele, PM. (1986). Development and Learning Process. A study of some aspects of Piaget psychology in relation with the didactics of mathematics. Groningen Utrecht: JB Wolters.

Weber, K. (2010). Mathematics' majors perceptions of conviction, validity, and proof. Mathematical Thinking and Learning 12, 306-336.

Weber, K. (2002). Student difficulty in constructing proofs: The need for strategic knowledge. Educational Studies in Mathematics. Dordrecht: Kluwer Academic Publishers. 48, 101-119. 